\title{
Association between GRK4 and DRD / gene polymorphisms and hypertension: a meta-analysis
}

This article was published in the following Dove Press journal:

Clinical Interventions in Aging

23 December 2015

Number of times this article has been viewed

\author{
He Zhang \\ Zhao-qing Sun \\ Shuang-shuang Liu \\ Li-na Yang
}

Department of Cardiology, Shengjing Hospital of China Medical University, Shenyang, People's Republic of China
Correspondence: He Zhang

Department of Cardiology, Shengjing Hospital of China Medical University, 36 Sanhao Street, Heping District, Shenyang, I I0004, People's Republic of China Email zhanghe_77@I63.com
Abstract: The role of GRK4 and DRDl genes in hypertension remains controversial. We performed a meta-analysis to determine whether GRK4 and DRD1 polymorphisms influence the risk of hypertension and examined the relationship between the genetic variances and the etiology of hypertension. Relevant case-control studies were retrieved by database searches and selected according to established inclusion criteria. Odds ratios (ORs) and 95\% confidence intervals (CIs) were calculated to evaluate the strength of the associations. Meta-regression, subgroup analysis, and sensitivity analysis were performed. A total of 15 articles containing 29 studies were finally included. In the dominant model, rs4532 locus of DRD1 gene was related to hypertension with a pooled OR of 1.353 ( $95 \% \mathrm{CI}=1.016-1.802, P=0.038)$. Subgroup analysis for ethnicity showed that rs 1024323 locus of GRK4 gene was associated with hypertension in Caucasians ( $\mathrm{OR}=1.826,95 \% \mathrm{CI}=1.215-2.745, P=0.004)$ but not in East Asians and Africans. Rs4532 locus was associated with hypertension in East Asians ( $\mathrm{OR}=1.833$, $95 \% \mathrm{CI}=1.415-2.376, P<0.001)$ but not in Caucasians. These data provide possible references for future case-control studies in hypertension.

Keywords: $G R K 4, D R D 1$, hypertension, meta-analysis, gene polymorphism

\section{Introduction}

Hypertension is a common complex disease affected by the mutual influence of multiple genetic and environmental factors. ${ }^{1,2}$ It is a major risk factor for many disorders, such as renal failure, stroke, and cardiovascular disease, ${ }^{3,4}$ and its heritability ranges from $30 \%$ to $50 \%{ }^{5,6}$ However, the definite genetic background of hypertension is difficult to determine and remains inconclusive. Molecular genetics research has been attempting to ascertain the inherited susceptible genes of hypertension. Numerous efforts have been concentrated on the abnormal renal handling of sodium chloride metabolism in the pathogenesis of hypertension, considering that the kidney plays an important role in the long-term control of blood pressure and is the major organ involved in the regulation of sodium homeostasis. ${ }^{7,8}$ Therefore, GRK4 and DRD1 genes have been assessed as the potential candidates.

GRKs are a seven-member family of serine/threonine protein kinases distinguished by their ability to specifically recognize, phosphorylate - and desensitize agonistactivated GPCRs. ${ }^{9}$ The sodium retention in hypertension is on account of increased sodium transport and/or deficiency to respond appropriately to signs that decrease sodium transport. ${ }^{10}$ The process of sodium transport is regulated by natriuretic and anti-natriuretic hormones, which exert their effects by GPCRs. Thus, GRK-mediated receptor phosphorylation is one of the well-distinguished mechanisms for GPCR desensitization. Interestingly, $G R K 4$, different from the other $G R K \mathrm{~s}$ in tissue distribution, is abundantly expressed in the kidney and seems to play a vital role in the regulation of 
sodium metabolism. ${ }^{11}$ GRK4 gene is located in chromosome 4 ( $4 \mathrm{p} 16.3)$ and four splice variants $(G R K 4 \alpha, \beta, \gamma, \delta)$ have been identified in humans. ${ }^{12}$ An increasing number of studies show that GRK4 gene is associated with hypertension and blood pressure in different ethnic populations. ${ }^{13-16}$ Among them, rs2960306 (R65L), rs1024323 (A142V), and rs1801058 (A486V) polymorphisms have attracted the most attention.

GRK4 mainly exerts its function by $D R D 1$ and its impaired coupling of $D R D 1$ and its G-protein effector enzyme complex has been linked to the dopamine-mediated sodium dysregulation. ${ }^{10}$ Likewise, $D R D 1$-deficient mice develop hypertension. ${ }^{17}$ Therefore, $D R D 1$ seems to be a risk factor in the development of hypertension, considering that dopaminergic and cholinergic neurotransmitters mainly regulate blood pressure by binding to their respective receptors. ${ }^{18}$ Many efforts have been made to screen the latent pathogenic variations of $D R D 1$ gene. Among them, rs4532 (A48G), localizing the promoter region, was found to be associated with hypertension in Japanese and Chinese. ${ }^{19,20}$ This single nucleotide polymorphism may affect the expression of $D R D 1$ by impacting micro RNA-mediated posttranscriptional regulation. ${ }^{21}$

Recently, many molecular epidemiological studies ${ }^{22-31}$ were performed to investigate the association between the four aforementioned loci and hypertension. Unfortunately, the results were conflicting or inconsistent, most likely due to small sample size, diverse genetic backgrounds, and potential confounding bias. Meta-analysis is a widely used statistical method in medical study, particularly for a subject being extensively investigated while controversial results are being reported. ${ }^{32}$ One meta-analysis was performed in 2012 to evaluate the association of three polymorphisms (rs2960306, rs1024323, and rs1801058) of GRK4 gene with hypertension. ${ }^{33}$ The pooled result showed that rs 1801058 was associated with hypertension in East Asians and Europeans and there was a significant association between rs2960306 and hypertension among Europeans. However, Liu and Xi's meta-analysis only included five studies and the literature search was updated in September 2011. Additionally, new molecular epidemiological studies have recently been conducted to investigate the role of GRK4 gene variations in the occurrence of hypertension in different populations and provide new evidences that were not included in the previous meta-analysis. Furthermore, the association of rs4532 polymorphism of DRD1 gene in the disease has not been clarified in the meta-analysis. Consequently, we carried out a meta-analysis of studies examining these single nucleotide polymorphisms to provide a more comprehensive assessment of the association of GRK4 and $D R D 1$ polymorphisms with hypertension.

\section{Materials and methods Identification and eligibility of relevant studies}

To identify studies eligible for inclusion in this meta-analysis, five online electronic databases (PubMed, Embase, and Web of Science in English; and China National Knowledge Infrastructure and Wanfang Database in Chinese) were searched (the last search update was August 2015). The following keywords were used in the literature search: G-protein coupled receptor kinase 4, GRK4, dopamine receptor 1, $D R D 1$, hypertension, and preeclampsia. Reference lists from retrieved articles and potentially relevant review articles were also hand-searched for additional studies. Studies met the following inclusion criteria: 1) case-control design, regardless of sample size, using hospital-based or population-based controls; 2) patients with hypertension; and 3) presented available allele or genotype frequencies. For duplicate publication, the most recent or largest articles were included. Study authors were contacted for additional details (eg, allele or genotype frequencies or sample characteristics) if we needed to retrieve additional data which were not stated in the original report.

\section{Data extraction}

Based on the inclusion criteria, two reviewers (He Zhang and Zhao-qing Sun) independently extracted the information from all qualified literatures. Disagreements were resolved through discussion until the two reviewers reached a consensus. The following data were extracted from each study: first author's last name, publication year, region, and counts of alleles and genotypes between cases and controls. To delineate potential moderating influences on the effects obtained from the case-control studies under consideration, we also included the following variables: 1) ethnicity of the sample population; 2) source of the controls; 3 ) mean age of the control and case group; 4) sex proportion; and 5) definition of hypertension and controls.

\section{Quality assessment}

Two authors (He Zhang and Shuang-shuang Liu) independently assessed the quality of the included studies according to the Newcastle-Ottawa Scale (www.ohri.ca/programs/ clinical epidemiology/oxfprd.asp). This scale consists of three components related to sample selection, comparability, and ascertainment of exposure. A score of 5 or more (maximum of 9) was regarded as "high quality"; studies with scores from 0 to 4 were considered "low quality". ${ }^{34}$ 


\section{Statistical analysis}

All statistical tests were two-sided, and $P<0.05$ was considered statistically significant. The meta-analysis was performed using Stata version 10.0 (Stata Corp LP, College Station, TX, USA). Hardy-Weinberg equilibrium (HWE) in the genotype distribution of controls was calculated again in our meta-analysis. The chi-square goodness of fit was used to test deviation from HWE.

The strength of the association between the target locus and hypertension was estimated by odds ratios (ORs) with 95\% confidence intervals (CIs). Pooled effect sizes across studies were performed by a random effects model. ${ }^{35}$ Overall pooled ORs were calculated using the allele contrast model, dominant model, and recessive model. Comparisons of $\mathrm{OR}_{1}$ (AA vs aa), $\mathrm{OR}_{2}$ (Aa vs aa) and $\mathrm{OR}_{3}$ (AA vs Aa) were explored with $\mathrm{A}$ as the risk allele. ${ }^{32}$ The aforementioned pairwise differences were used to determine the most appropriate genetic model. If $\mathrm{OR}_{1}=\mathrm{OR}_{3} \neq 1$ and $\mathrm{OR}_{2}=1$, then a recessive model was selected. If $\mathrm{OR}_{1}=\mathrm{OR}_{2} \neq 1$ and $\mathrm{OR}_{3}=1$, then a dominant model was selected. If $\mathrm{OR}_{2}=1 / \mathrm{OR}_{3} \neq 1$ and $\mathrm{OR}_{1}=1$, then a complete overdominant model was selected. If $\mathrm{OR}_{1}>\mathrm{OR}_{2}>1$ and $\mathrm{OR}_{1}>\mathrm{OR}_{3}>1$ (or $\mathrm{OR}_{1}<\mathrm{OR}_{2}<1$ and $\left.\mathrm{OR}_{1}<\mathrm{OR}_{3}<1\right)$, then a codominant model was selected. ${ }^{36}$

The degree of heterogeneity was determined by Q-statistic, and $P$-value and $I^{2}$ were used to evaluate the heterogeneity among different studies. ${ }^{37-39}$ Subgroup analysis was performed by ethnicity (East Asian, African, and Caucasian) and source of controls (hospital-based and population-based). Meta-regression was employed to explore the potential sources of heterogeneity including publication date, ethnicity, source of controls, mean age of control and case group, and sex. An estimate of publication bias was assessed by funnel plot and Egger's test. ${ }^{40}$ Sensitivity analysis was also performed to weight the potential influences of every single study on the pooled effect size. ${ }^{41}$

\section{Results}

After the removal of overlapping articles and those that did not meet the inclusion criteria, a total of 15 articles including 29 studies were finally included in our metaanalysis. ${ }^{19,20,22-31,42-44}$ The main characteristics of the included studies were presented in Table 1. For GRK4 gene, seven studies with 1,704 cases and 1,705 controls dealt with rs 1801058 , six studies with 1,598 cases and 1,611 controls dealt with rs2960306, and nine studies with 1,973 cases and 1,946 controls dealt with rs1024323. For DRD1 gene, seven studies with 2,083 cases and 1,383 controls dealt with rs4532. Of the total 29 studies, Sanada et al' study concerning rs 1801058 and rs2960306 presented significant deviation from HWE. ${ }^{19}$ In light of Newcastle-Ottawa Scale, eleven articles are high quality and four articles are low quality. Genotype and allele frequencies, HWE, and sample size information are described in Tables $2-5$.

\section{Quantitative synthesis and heterogeneity analysis \\ Association of rs I80I058 locus (GRK4 A486V) with hypertension}

We analyzed seven studies with 1,704 cases and 1,705 controls dealt with the association between rs1801058

Table I Baseline characteristics of qualified studies in this meta-analysis

\begin{tabular}{|c|c|c|c|c|c|c|c|c|}
\hline Author & Year & Country & Ethnicity & $\begin{array}{l}\text { Controls } \\
\text { source }\end{array}$ & $\begin{array}{l}\text { Mean age of } \\
\text { control group } \\
\text { (years) }\end{array}$ & $\begin{array}{l}\text { Mean age of } \\
\text { case group } \\
\text { (years) }\end{array}$ & $\begin{array}{l}\text { Sex } \\
\text { index }\end{array}$ & $\begin{array}{l}\text { NOS } \\
\text { scores }\end{array}$ \\
\hline Sato et $\mathrm{a}^{43}$ & 2000 & Japan & East Asians & Population-based & 49.00 & 49.00 & 1.69 & 6 \\
\hline Bengra et $\mathrm{al}^{22}$ & 2002 & Italy & Caucasians & Hospital-based & - & - & - & 3 \\
\hline Yuan et $\mathrm{al}^{42}$ & 2002 & People's Republic of China & East Asians & Hospital-based & 56.48 & 57.50 & 0.74 & 5 \\
\hline Beige et $\mathrm{al}^{30}$ & 2004 & Canada & Caucasians & Hospital-based & 30.70 & 54.60 & 1.50 & 7 \\
\hline Speirs et $\mathrm{al}^{24}$ & 2004 & Australia and UK & Caucasians & Population-based & 47.00 & 54.00 & 1.99 & 8 \\
\hline Williams et $\mathrm{al}^{23}$ & 2004 & Ghana & Africans & Hospital-based & - & - & - & 5 \\
\hline Wang et $\mathrm{al}^{25}$ & 2006 & People's Republic of China & East Asians & Population-based & 53.51 & 53.57 & I.0I & 6 \\
\hline Xu et $\mathrm{al}^{20}$ & 2006 & People's Republic of China & East Asians & Hospital-based & 49.28 & 50.19 & 0.88 & 6 \\
\hline Cao et $\mathrm{al}^{28}$ & 2007 & People's Republic of China & East Asians & Hospital-based & - & - & - & 3 \\
\hline Martinez Cantarin et $\mathrm{a}^{26}$ & 2010 & USA & Africans & Population-based & 36.00 & 40.00 & 0.61 & 9 \\
\hline Sun and Zhang ${ }^{27}$ & 2010 & People's Republic of China & East Asians & Hospital-based & 30.36 & 29.13 & - & 6 \\
\hline Orun et $\mathrm{al}^{44}$ & 2011 & Turkey & Caucasians & Population-based & 36.70 & 58.12 & 2.26 & 5 \\
\hline Cipolletta et $\mathrm{al}^{31}$ & 2012 & Italy & Caucasians & Hospital-based & - & 56.01 & - & 4 \\
\hline Kimura et $\mathrm{al}^{29}$ & 2012 & Brazil & Africans & Population-based & 32.00 & 55.70 & 1.14 & 4 \\
\hline Sanada et al $^{19}$ & 2015 & Japan & East Asians & Population-based & 57.50 & 56.20 & 0.86 & 8 \\
\hline
\end{tabular}

Note: Sex index = (female cases/male cases) $/($ female controls $/$ male controls).

Abbreviation: NOS, Newcastle-Ottawa Scale. 
Table 2 Distribution of genotype and allele frequencies of the GRK4 rs I80 I058 (GRK4 A486V) locus

\begin{tabular}{|c|c|c|c|c|c|c|c|c|c|c|c|c|c|}
\hline \multirow[t]{3}{*}{ Author } & \multicolumn{6}{|c|}{ Genotype distribution } & \multirow[t]{3}{*}{$P_{\text {HWE }}$} & \multicolumn{4}{|c|}{ Allele frequency } & \multicolumn{2}{|c|}{ Sample size } \\
\hline & \multicolumn{3}{|c|}{ Cases, $\mathbf{n}$} & \multicolumn{3}{|c|}{ Controls, $n$} & & \multicolumn{2}{|c|}{ Cases, \% } & \multicolumn{2}{|c|}{ Controls, \% } & \multirow[t]{2}{*}{ Case } & \multirow[t]{2}{*}{ Control } \\
\hline & GG & GT & TT & GG & GT & TT & & G & $\mathbf{T}$ & $\mathbf{G}$ & $\mathbf{T}$ & & \\
\hline Bengra et $\mathrm{a}^{22}$ & 24 & 23 & 13 & 28 & 25 & 7 & 0.6966 & 59.2 & 40.8 & 67.5 & 32.5 & 60 & 60 \\
\hline Williams et $\mathrm{a}^{23}$ & 31 & 64 & 29 & 12 & 25 & 14 & 0.8972 & 50.8 & 49.2 & 48.0 & 52.0 & 124 & 51 \\
\hline Speirs et $\mathrm{al}^{24}$ & 57 & 77 & 26 & 117 & 166 & 29 & 0.0058 & 59.7 & 40.3 & 64.1 & 35.9 & 160 & 312 \\
\hline Wang et $\mathrm{a}^{25}$ & 400 & 97 & 6 & 372 & 109 & 9 & 0.7575 & 89.2 & 10.8 & 87.0 & 13.0 & 503 & 490 \\
\hline Martinez Cantarin et $\mathrm{a}^{26}$ & 49 & 90 & 26 & 60 & 115 & 31 & 0.0459 & 57.0 & 43.0 & 57.0 & 43.0 & 165 & 206 \\
\hline Sanada et al ${ }^{19}$ & 424 & 153 & 10 & 433 & 45 & 5 & 0.0036 & 85.3 & 14.7 & 94.3 & 5.7 & 587 & 483 \\
\hline Sun and Zhang ${ }^{27}$ & 31 & 63 & 11 & 35 & 53 & 15 & 0.4809 & 59.5 & 40.5 & 59.7 & 40.3 & 105 & 103 \\
\hline
\end{tabular}

Note: $P_{\text {HWE }}$ represents the $P$-value of Hardy-Weinberg equilibrium test in the genotype distribution of controls.

Table 3 Distribution of genotype and allele frequencies of the GRK4 rs2960306 (GRK4 R65L) locus

\begin{tabular}{|c|c|c|c|c|c|c|c|c|c|c|c|c|c|}
\hline \multirow[t]{3}{*}{ Author } & \multicolumn{6}{|c|}{ Genotype distribution } & \multirow[t]{3}{*}{$\boldsymbol{P}_{\mathrm{HWE}}$} & \multicolumn{4}{|c|}{ Allele frequency } & \multicolumn{2}{|c|}{ Sample size } \\
\hline & \multicolumn{3}{|c|}{ Cases, $\mathbf{n}$} & \multicolumn{3}{|c|}{ Controls, n } & & \multicolumn{2}{|c|}{ Cases, \% } & \multicolumn{2}{|c|}{ Controls, \% } & \multirow[t]{2}{*}{ Case } & \multirow[t]{2}{*}{ Contro } \\
\hline & CC & CT & TT & CC & CT & TT & & C & $\mathbf{T}$ & C & $\mathbf{T}$ & & \\
\hline Bengra et $\mathrm{al}^{22}$ & 27 & 22 & 11 & 28 & 25 & 7 & 0.6966 & 63.3 & 36.7 & 67.5 & 32.5 & 60 & 60 \\
\hline Williams et $\mathrm{al}^{23}$ & 12 & 62 & 51 & 8 & 23 & 20 & 0.7470 & 34.4 & 65.6 & 38.2 & 61.8 & 125 & 51 \\
\hline Speirs et $\mathrm{al}^{24}$ & 60 & 84 & 24 & 76 & 92 & 21 & 0.3818 & 60.7 & 39.3 & 64.6 & 35.4 & 160 & 312 \\
\hline Wang et $\mathrm{al}^{25}$ & 344 & 143 & 16 & 309 & 156 & 25 & 0.3621 & 82.6 & 17.4 & 79.0 & 21.0 & 503 & 490 \\
\hline Martinez Cantarin et $\mathrm{al}^{26}$ & 53 & 82 & 27 & 73 & 111 & 30 & 0.2359 & 58.0 & 42.0 & 60.0 & 40.0 & 162 & 214 \\
\hline Sanada et al ${ }^{19}$ & 361 & 204 & 23 & 393 & 79 & 12 & 0.0018 & 78.7 & 21.3 & 89.4 & 10.6 & 588 & 484 \\
\hline
\end{tabular}

Note: $P_{\text {HWE }}$ represents the $P$-value of Hardy-Weinberg equilibrium test in the genotype distribution of controls.

Table 4 Distribution of genotype and allele frequencies of the GRK4 rs 1024323 (GRK4 AI42V) locus

\begin{tabular}{|c|c|c|c|c|c|c|c|c|c|c|c|c|c|}
\hline \multirow[t]{3}{*}{ Author } & \multicolumn{6}{|c|}{ Genotype distribution } & \multirow[t]{3}{*}{$P_{\text {HWE }}$} & \multicolumn{4}{|c|}{ Allele frequency } & \multicolumn{2}{|c|}{ Sample size } \\
\hline & \multicolumn{3}{|c|}{ Cases, $\mathbf{n}$} & \multicolumn{3}{|c|}{ Controls, $n$} & & \multicolumn{2}{|c|}{ Cases, \% } & \multicolumn{2}{|c|}{ Controls, \% } & \multirow[t]{2}{*}{ Case } & \multirow[t]{2}{*}{ Contro } \\
\hline & CC & CT & TT & CC & CT & TT & & C & $\mathbf{T}$ & C & $\mathbf{T}$ & & \\
\hline Bengra et $\mathrm{al}^{22}$ & 15 & 29 & 16 & 25 & 26 & 9 & 0.6039 & 49.2 & 50.8 & 63.3 & 36.7 & 60 & 60 \\
\hline Williams et $\mathrm{a}^{23}$ & 99 & 21 & 4 & 42 & 9 & 0 & 0.4895 & 88.3 & 11.7 & 91.2 & 8.8 & 124 & 51 \\
\hline Speirs et $\mathrm{al}^{24}$ & 31 & 84 & 30 & 79 & 134 & 35 & 0.0683 & 50.3 & 49.7 & 58.9 & 41.1 & 145 & 248 \\
\hline Wang et $\mathrm{a}^{25}$ & 169 & 218 & 116 & 96 & 226 & 168 & 0.2055 & 55.3 & 44.7 & 42.7 & 57.3 & 503 & 490 \\
\hline Martinez Cantarin et $\mathrm{a}^{26}$ & 128 & 37 & 3 & 140 & 65 & 5 & 0.4248 & 87.2 & 12.8 & 82.1 & 17.9 & 168 & 210 \\
\hline Sanada et al ${ }^{19}$ & 145 & 286 & 157 & $|8|$ & 227 & 77 & 0.6788 & 49.0 & 51.0 & 60.7 & 39.3 & 588 & 485 \\
\hline Sun and Zhang ${ }^{27}$ & 68 & 34 & 3 & 72 & 29 & 2 & 0.6376 & 81.0 & 19.1 & 84.0 & 16.0 & 105 & 103 \\
\hline Cao et $\mathrm{al}^{28}$ & 19 & 50 & 33 & 28 & 48 & 17 & 0.6510 & 43.1 & 56.9 & 55.9 & 44.1 & 102 & 93 \\
\hline Kimura et $\mathrm{al}^{29}$ & 98 & 72 & 8 & 117 & 72 & 17 & 0.2200 & 75.3 & 24.7 & 74.3 & 25.7 & 178 & 206 \\
\hline
\end{tabular}

Note: $P_{\text {HWE }}$ represents the $P$-value of Hardy-Weinberg equilibrium test in the genotype distribution of controls.

Table 5 Distribution of genotype and allele frequencies of the DRD / rs4532 locus

\begin{tabular}{|c|c|c|c|c|c|c|c|c|c|c|c|c|c|}
\hline \multirow[t]{3}{*}{ Author } & \multicolumn{6}{|c|}{ Genotype distribution } & \multirow[t]{3}{*}{$P_{\text {HWE }}$} & \multicolumn{4}{|c|}{ Allele frequency } & \multicolumn{2}{|c|}{ Sample size } \\
\hline & \multicolumn{3}{|c|}{ Cases, $\mathbf{n}$} & \multicolumn{3}{|c|}{ Controls, n } & & \multicolumn{2}{|c|}{ Cases, \% } & \multicolumn{2}{|c|}{ Controls, $\%$} & \multirow[t]{2}{*}{ Case } & \multirow[t]{2}{*}{ Contro } \\
\hline & AA & GA & GG & AA & GA & GG & & $\mathbf{A}$ & $\mathbf{G}$ & $\mathbf{A}$ & $\mathbf{G}$ & & \\
\hline Sanada et al ${ }^{19}$ & 6 & 100 & 482 & 8 & 107 & 368 & 0.9447 & 9.5 & 90.5 & 12.7 & 87.3 & 588 & 483 \\
\hline Beige et $\mathrm{a}^{30}$ & 188 & 253 & 52 & 78 & 107 & 24 & 0.1601 & 63.8 & 36.2 & 62.9 & 37.1 & 493 & 209 \\
\hline Cipolletta et $\mathrm{a}^{31}$ & 33 & 126 & 94 & 12 & 51 & 36 & 0.3464 & 37.9 & 62.1 & 37.9 & 62.1 & 253 & 100 \\
\hline Xu et $a^{20}$ & 211 & 105 & 14 & 147 & 44 & 4 & 0.7410 & 79.9 & 20.2 & 86.7 & 13.3 & 330 & 195 \\
\hline Yuan et $\mathrm{a}^{42}$ & 111 & 68 & 8 & 115 & 38 & 3 & 0.9460 & 77.5 & 22.5 & 85.9 & 14.1 & 187 & 156 \\
\hline Sato et $\mathrm{a}^{43}$ & 93 & 35 & 3 & 113 & 23 & 0 & 0.2814 & 84.4 & 15.6 & 91.5 & 8.5 & $13 \mid$ & 136 \\
\hline Orun et $\mathrm{a}^{44}$ & 47 & 36 & 18 & 46 & 44 & 14 & 0.5051 & 64.4 & 35.6 & 65.4 & 34.6 & 101 & 104 \\
\hline
\end{tabular}

Note: $P_{\text {HWE }}$ represents the $P$-value of Hardy-Weinberg equilibrium test in the genotype distribution of controls. 
Table 6 Summarized ORs with 95\% Cls for the association of GRK4 and DRD / polymorphisms with hypertension

\begin{tabular}{|c|c|c|c|c|c|c|c|c|c|}
\hline Polymorphism & Genetic model & $\mathbf{n}$ & Statistical model & OR & $95 \% \mathrm{Cl}$ & $P_{\mathrm{z}}$ & $I^{2}(\%)$ & $P_{\mathrm{h}}$ & $P_{\mathrm{e}}$ \\
\hline \multicolumn{10}{|c|}{ RsI80I058 (A486V) } \\
\hline & Allele contrast & 7 & Random & 1.203 & $0.858-1.688$ & 0.284 & 85.4 & $<0.001$ & 0.886 \\
\hline & Homozygous codominant & 7 & Random & 1.213 & $0.856-1.719$ & 0.277 & 15.1 & 0.315 & 0.716 \\
\hline & Heterozygous codominant & 7 & Random & 1.223 & $0.760-1.969$ & 0.407 & 85.6 & $<0.001$ & 0.848 \\
\hline & Dominant & 7 & Random & 1.243 & $0.789-1.958$ & 0.349 & 85.5 & $<0.001$ & 0.864 \\
\hline & Recessive & 7 & Random & 1.155 & $0.815-1.639$ & 0.418 & 28.5 & 0.211 & 0.687 \\
\hline \multicolumn{10}{|l|}{ Rs2960306 (R65L) } \\
\hline & Allele contrast & 6 & Random & 1.219 & $0.85 \mathrm{I}-\mathrm{I} .747$ & 0.279 & 87.2 & $<0.001$ & 0.940 \\
\hline & Homozygous codominant & 6 & Random & 1.280 & $0.858-1.911$ & 0.226 & 39.7 & 0.141 & 0.430 \\
\hline & Heterozygous codominant & 6 & Random & 1.278 & $0.768-2.126$ & 0.344 & 87.3 & $<0.001$ & 0.882 \\
\hline & Dominant & 6 & Random & 1.304 & $0.793-2.145$ & 0.295 & 88.0 & $<0.001$ & 0.971 \\
\hline & Recessive & 6 & Random & 1.144 & $0.858-1.525$ & 0.360 & 8.3 & 0.363 & 0.504 \\
\hline \multicolumn{10}{|c|}{ RsI024323 (AI42V) } \\
\hline & Allele contrast & 9 & Random & 1.161 & $0.830-1.625$ & 0.383 & 90.0 & $<0.001$ & 0.620 \\
\hline & Homozygous codominant & 9 & Random & 1.413 & $0.67 \mid-2.975$ & 0.362 & 89.0 & $<0.001$ & 0.609 \\
\hline & Heterozygous codominant & 9 & Random & 1.120 & $0.796-1.577$ & 0.515 & 77.7 & $<0.001$ & 0.630 \\
\hline & Dominant & 9 & Random & 1.190 & $0.785-1.805$ & 0.413 & 86.7 & $<0.001$ & 0.581 \\
\hline & Recessive & 9 & Random & 1.257 & $0.745-2.123$ & 0.391 & 82.3 & $<0.001$ & 0.593 \\
\hline \multicolumn{10}{|l|}{ Rs4532 } \\
\hline & Allele contrast & 7 & Random & 1.303 & $1.055-1.610$ & 0.014 & 62.0 & 0.015 & 0.156 \\
\hline & Homozygous codominant & 7 & Random & I.27I & $0.886-1.824$ & 0.192 & 8.3 & 0.365 & 0.002 \\
\hline & Heterozygous codominant & 7 & Random & 1.287 & $0.976-1.696$ & 0.073 & 44.8 & 0.093 & 0.947 \\
\hline & Dominant & 7 & Random & 1.353 & $1.016-1.802$ & 0.038 & 52.2 & 0.051 & 0.751 \\
\hline & Recessive & 7 & Random & 1.282 & $1.039-1.582$ & 0.021 & 0.0 & 0.445 & 0.289 \\
\hline
\end{tabular}

Notes: $\mathrm{n}$, the number of studies; $P_{\mathrm{z}}, P$-value for association test; $P_{\mathrm{h}}, P$-value for heterogeneity test; $P$, $P$-value for publication bias test.

Abbreviations: ORs, odds ratios; $\mathrm{Cls}$, confidence intervals.

and hypertension. The dominant model was determined according to the principle of genetic model selection (Table 6, Figure 1). ${ }^{36,45}$ The summary results indicated that there was no association between rs1801058 locus and the occurrence of hypertension. The pooled OR using random effects model was $1.243(95 \% \mathrm{CI}=0.789-1.958$, $P=0.349)$. Subgroup analysis for ethnicity indicated that the locus was not associated with hypertension among East Asians, Africans, and Caucasians (Table 7). Moreover, no association between rs 1801058 locus and hypertension was

\begin{tabular}{|c|c|c|}
\hline Study ID & OR $(95 \% \mathrm{CI})$ & $\%$ weight \\
\hline Bengra et $\mathrm{al}^{22}$ & $1.31(0.64-2.71)$ & 12.12 \\
\hline Speirs et al ${ }^{24}$ & $1.08(0.73-1.61)$ & 15.45 \\
\hline Williams et $a^{23}$ & $0.92(0.43-1.98)$ & 11.71 \\
\hline Wang et $\mathrm{al}^{25}$ & $0.81(0.60-1.10)$ & 16.27 \\
\hline Martinez Cantarin et $\mathrm{al}^{26}$ & $0.97(0.62-1.52)$ & 14.95 \\
\hline Sun and Zhang ${ }^{27}$ & $1.23(0.68-2.21)$ & 13.57 \\
\hline Sanada et al ${ }^{19}$ & $\rightarrow 3.33(2.36-4.70)$ & 15.91 \\
\hline Overall $\left(I^{2}=85.5 \%, P=0.000\right)$ & $1.24(0.79-1.96)$ & 100.00 \\
\hline
\end{tabular}

Figure I Forest plot of the association between GRK4 rs I80I058 (GRK4 A486V) locus and hypertension in dominant model (GT + TT vs GG).

Note: Weights are from random effects analysis.

Abbreviations: $\mathrm{OR}$, odds ratio; $\mathrm{Cl}$, confidence interval. 
Table 7 Stratified analysis for the association of GRK4 and DRD I polymorphisms with hypertension under dominant model

\begin{tabular}{|c|c|c|c|c|c|c|c|c|}
\hline Polymorphism & Subgroup type & Subgroup & $\mathbf{n}$ & OR & $95 \% \mathrm{Cl}$ & $P_{\mathrm{z}}$ & $I^{2}(\%)$ & $P_{\mathrm{h}}$ \\
\hline \multicolumn{9}{|c|}{ RsI80I058 (A486V) } \\
\hline & Ethnicity & Caucasians & 2 & 1.133 & $0.800-1.604$ & $0.48 I$ & 0.0 & 0.650 \\
\hline & & Africans & 2 & 0.960 & $0.652-1.414$ & 0.836 & 0.0 & 0.907 \\
\hline & & East Asians & 3 & 1.496 & $0.569-3.931$ & 0.414 & 94.6 & $<0.001$ \\
\hline & Source of controls & Hospital-based & 3 & 1.162 & $0.786-1.718$ & 0.451 & 0.0 & 0.782 \\
\hline & & Population-based & 4 & 1.302 & $0.663-2.557$ & 0.443 & 92.6 & $<0.001$ \\
\hline \multicolumn{9}{|l|}{ Rs2960306 (R65L) } \\
\hline & Ethnicity & Caucasians & 2 & 1.172 & $0.811-1.694$ & 0.399 & 0.0 & 0.771 \\
\hline & & Africans & 2 & 1.158 & $0.780-1.719$ & 0.466 & 0.0 & 0.354 \\
\hline & & East Asians & 2 & 1.462 & $0.435-4.911$ & 0.539 & 97.5 & $<0.001$ \\
\hline & Source of controls & Hospital-based & 2 & 1.276 & $0.718-2.269$ & 0.406 & 0.0 & 0.420 \\
\hline & & Population-based & 4 & 1.294 & $0.693-2.415$ & 0.419 & 92.7 & $<0.001$ \\
\hline \multicolumn{9}{|l|}{ RsI 024323 (AI42V) } \\
\hline & Ethnicity & Caucasians & 2 & 1.826 & $1.215-2.745$ & 0.004 & 0.0 & 0.636 \\
\hline & & Africans & 3 & 0.885 & $0.590-1.325$ & 0.552 & 43.6 & 0.170 \\
\hline & & East Asians & 4 & 1.182 & $0.539-2.590$ & 0.677 & 93.7 & $<0.001$ \\
\hline & Source of controls & Hospital-based & 4 & 1.546 & $1.092-2.190$ & 0.014 & 0.0 & 0.600 \\
\hline & & Population-based & 5 & 1.000 & $0.556-1.800$ & 0.999 & 92.5 & $<0.001$ \\
\hline \multicolumn{9}{|l|}{ Rs4532 } \\
\hline & Ethnicity & Caucasians & 3 & 0.946 & $0.726-1.233$ & 0.684 & 0.0 & 0.981 \\
\hline & & East Asians & 4 & 1.833 & $1.415-2.376$ & $<0.001$ & 0.0 & 0.967 \\
\hline & Source of controls & Hospital-based & 4 & 1.337 & $0.913-1.960$ & 0.136 & 65.4 & 0.034 \\
\hline & & Population-based & 3 & I.391 & $0.80 I-2.4 I 6$ & $0.24 I$ & 48.0 & 0.146 \\
\hline
\end{tabular}

Notes: $\mathrm{n}$, the number of studies; $P_{\mathrm{z}}, P$-value for association test; $P_{\mathrm{h}}, P$-value for heterogeneity test. Abbreviations: $\mathrm{OR}$, odds ratio; $\mathrm{Cl}$, confidence interval.

observed when subgroup analysis for source of controls was conducted. Significant heterogeneity was observed, thus a meta-regression was conducted subsequently to explore the heterogeneity sources. However, the results of meta-regression indicated that ethnicity $(P=0.346)$, source of controls ( $P=0.776)$, age of the control group $(P=0.285)$, age of the case group $(P=0.200)$, and sex index $(P=0.956)$ had no statistical significance except for publication date $(P=0.020)$.

\section{Association of rs2960306 locus (GRK4 R65L) with hypertension}

We analyzed six studies with 1,598 cases and 1,611 controls dealt with the association between rs2960306 and hypertension. The dominant model was determined according to the principle of genetic model selection (Table 6, Figure 2). The summary results indicated that there was no association between rs2960306 locus and the occurrence of hypertension. The pooled OR using random effects model was 1.304 (95\% CI $=0.793-2.145, P=0.295)$. Subgroup analysis for ethnicity indicated that the locus was not associated with hypertension among East Asians, Africans, and Caucasians (Table 7). Moreover, no association between rs2960306 locus and hypertension was observed when subgroup analysis for source of controls was conducted. Significant heterogeneity was observed, thus a meta-regression was conducted subsequently to explore the heterogeneity sources. However, the results of meta-regression indicated that publication date $(P=0.063)$, ethnicity $(P=0.786)$, source of controls ( $P=0.963)$, age of the control group $(P=0.469)$, age of the case group $(P=0.236)$, and sex index $(P=0.757)$ had no statistical significance.

\section{Association of rs 1024323 locus (GRK4 AI42V) with hypertension}

We analyzed nine studies with 1,973 cases and 1,946 controls dealt with the association between rs1024323 and hypertension. The dominant model was determined according to the principle of genetic model selection (Table 6, Figure 3). The summary results indicated that there was no association between rs 1024323 locus and the occurrence of hypertension. The pooled OR using random effects model was 1.190 (95\% $\mathrm{CI}=0.785-1.805, P=0.413$ ). Subgroup analysis for ethnicity indicated that the locus was associated with hypertension in Caucasians ( $\mathrm{OR}=1.826,95 \% \mathrm{CI}=1.215-2.745, P=0.004$ ) but not in East Asians and Africans (Table 7). Moreover, the association between rs 1024323 locus and hypertension was observed when subgroup analysis for source of controls 


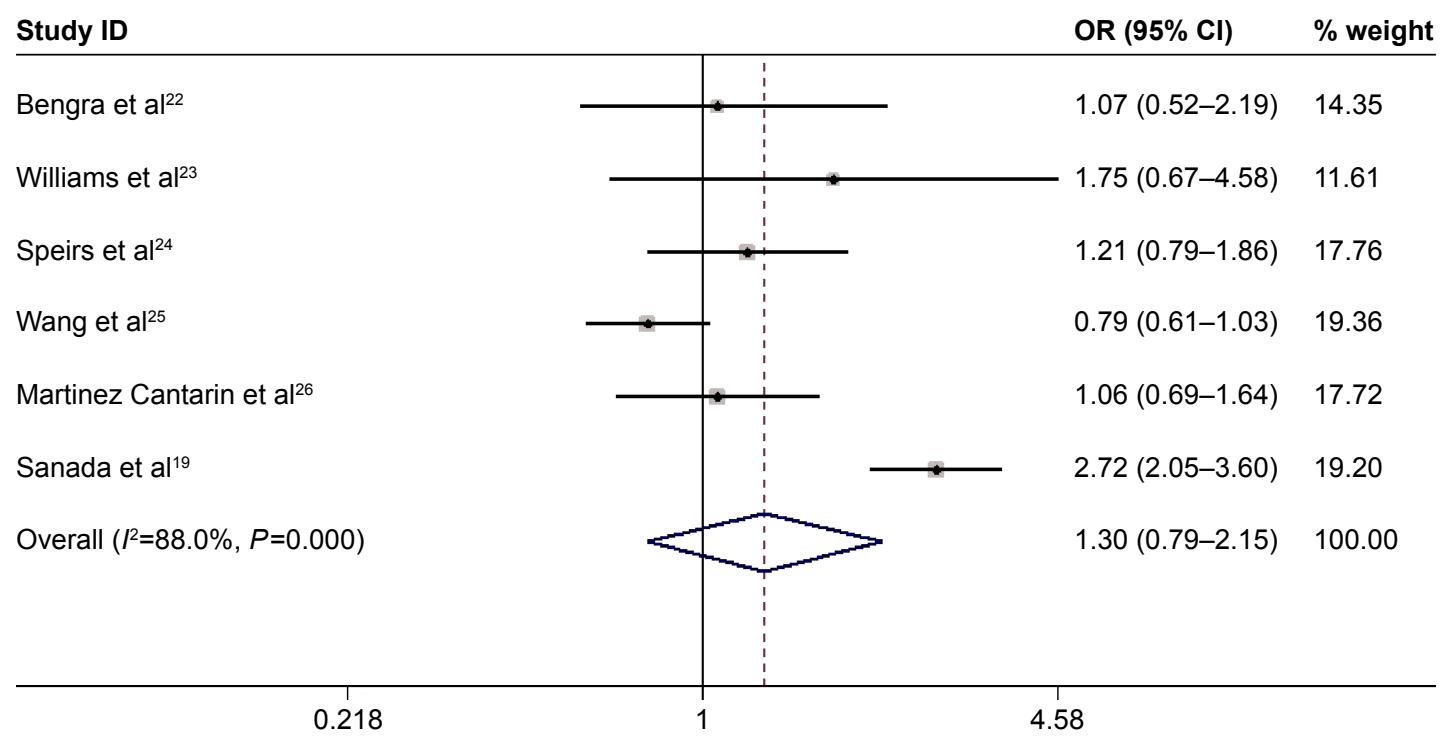

Figure 2 Forest plot of the association between GRK4 rs2960306 (GRK4 R65L) locus and hypertension in dominant model (CT + TT vs CC).

Note: Weights are from random effects analysis.

Abbreviations: $\mathrm{OR}$, odds ratio; $\mathrm{Cl}$, confidence interval.

was conducted (hospital-based subgroup: OR $=1.546,95 \%$ $\mathrm{CI}=1.092-2.190, P=0.014)$. Significant heterogeneity was observed, thus a meta-regression was conducted subsequently to explore the heterogeneity sources. However, the results of meta-regression indicated that publication date $(P=0.913)$, ethnicity $(P=0.640)$, source of controls $(P=0.234)$, age of the control group $(P=0.442)$, age of the case group $(P=0.917)$, and sex index $(P=0.674)$ had no statistical significance.

\section{Association of rs 4532 locus with hypertension}

We analyzed seven studies with 2,083 cases and 1,383 controls dealt with the association between rs4532 and hypertension. The dominant model was determined according to the principle of genetic model selection (Table 6, Figure 4). The summary results indicated that there was an association between rs4532 locus and the occurrence of hypertension. The pooled OR using random effects model was 1.353
Study ID

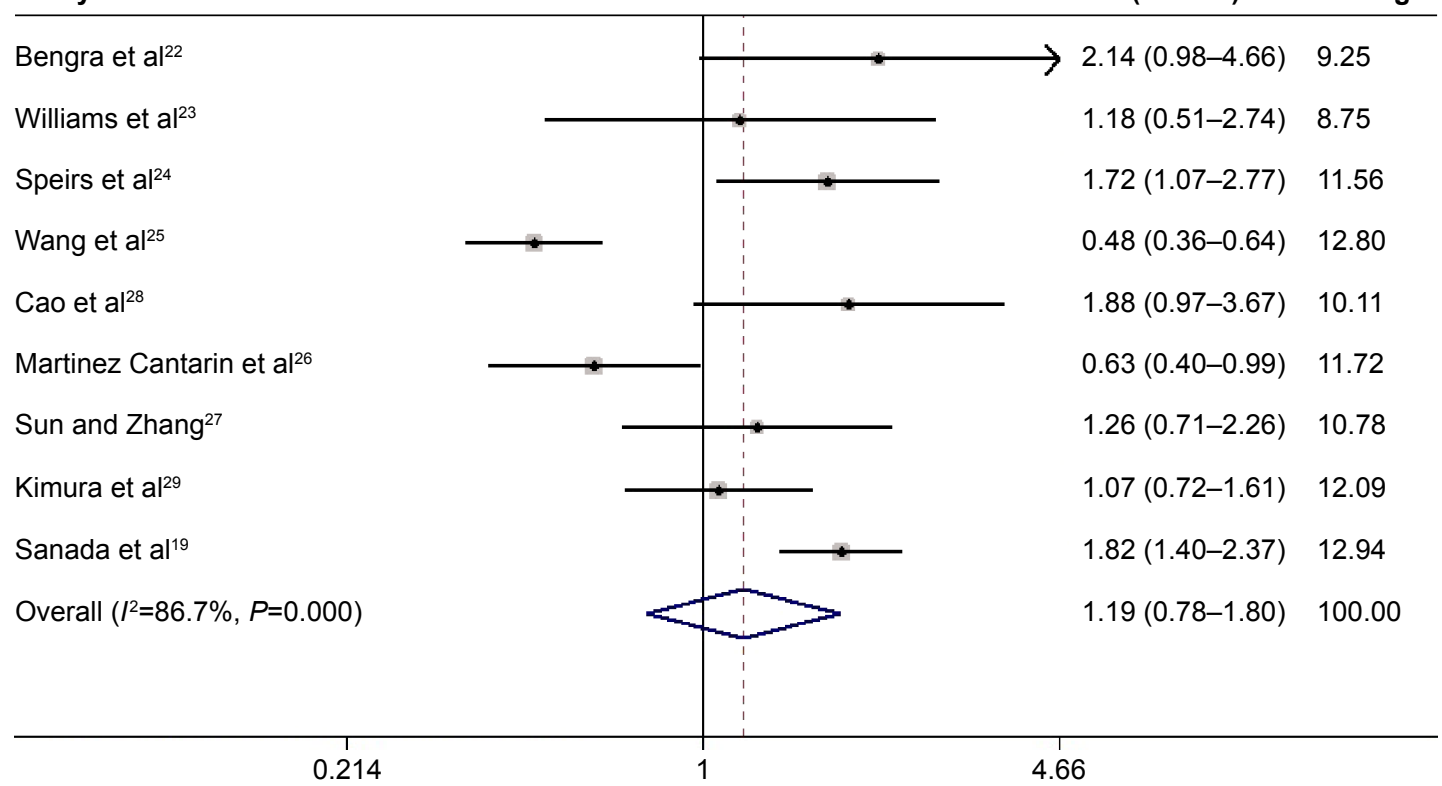

Figure 3 Forest plot of the association between GRK4 rs 1024323 (GRK4 AI42V) locus and hypertension in dominant model (CT + TT vs CC). Note: Weights are from random effects analysis.

Abbreviations: $\mathrm{OR}$, odds ratio; $\mathrm{Cl}$, confidence interval. 


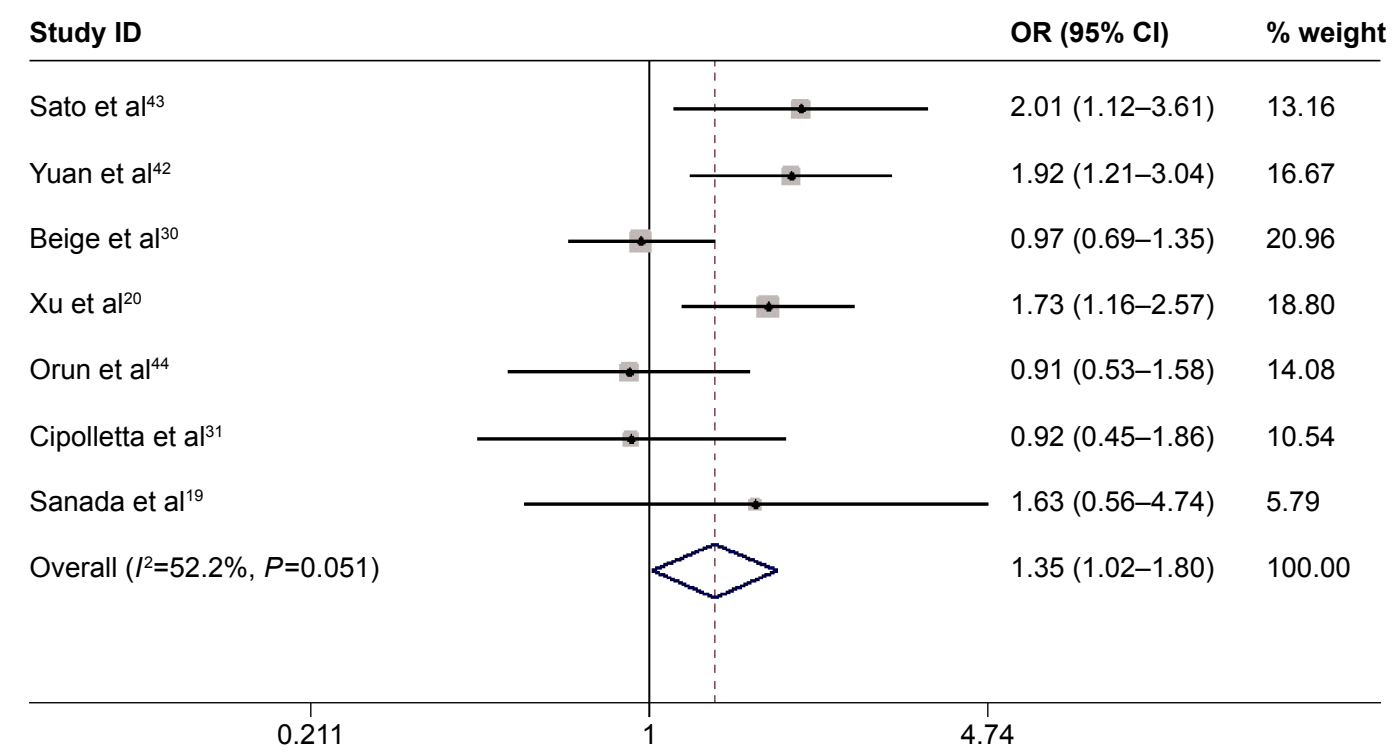

Figure 4 Forest plot of the association between DRD/ rs4532 locus and hypertension in dominant model (GA + GG vs AA). Note: Weights are from random effects analysis.

Abbreviations: $\mathrm{OR}$, odds ratio; $\mathrm{Cl}$, confidence interval.

(95\% CI $=1.016-1.802, P=0.038)$. Subgroup analysis for ethnicity indicated that the locus was associated with hypertension in East Asians ( $\mathrm{OR}=1.833,95 \% \mathrm{CI}=1.415-2.376$, $P<0.001$ ) but not in Caucasians (Table 7). Moreover, no association between rs 4532 locus and hypertension was observed when subgroup analysis for source of controls was conducted. Significant heterogeneity was observed, thus a meta-regression was conducted subsequently to explore the heterogeneity sources. However, the results of meta-regression indicated that publication date $(P=0.185)$, source of controls $(P=0.911)$, age of the case group $(P=0.240)$, and sex index $(P=0.082)$ had no statistical significance except for ethnicity $(P<0.001)$ and age of the control group ( $P=0.002)$.

\section{Sensitivity analysis}

Sensitivity analysis was carried out for each meta-analysis to address the influence of each study. Corresponding pooled ORs showed no significant change when one study was omitted at a time from each meta-analysis, implying that the results were stable and reliable.

\section{Publication bias}

A funnel plot was used to analyze the potential publication bias (Figure 5). Egger's test was performed to provide the statistical evidence for funnel plot symmetry and the result did not show any evidence of publication bias.

\section{Discussion}

The present meta-analysis included 15 articles containing 29 studies that investigated the association between GRK4 and DRDI gene polymorphism and the occurrence of hypertension. Overall, our meta-analytical results provided evidences that rs1024323 (GRK4 A142V) and rs4532 loci were associated with hypertension in Caucasians and East Asians, respectively. Subgroup analysis stratified by ethnicity and source of controls further explored the distribution disequilibrium of cases and controls. Sensitivity analysis and the examination of publication bias strengthened the validity of the results.

Previous studies reported the association between the polymorphisms of GRK4 gene and hypertension. ${ }^{13-16}$ Additionally, one meta-analysis has been done to investigate the association of GRK4 polymorphisms with hypertension, which suggested that rs1801058 (GRK4 $\mathrm{A} 486 \mathrm{~V}$ ) and rs2960306 (GRK4 R65L) loci were associated with hypertension. ${ }^{33}$ However, our results observed that rs1024323 and rs4532, rather than rs1801058 and rs2960306, were related to hypertension. To some extent, the current meta-analysis possessed several advantages over the previous study with respect to the following points. First, we included the recent published studies concerned with the association between GRK4 polymorphism and the occurrence of hypertension, which could provide more credibility for the final results. Second, besides stratified analyses by ethnicity and source of controls, we further performed meta-regression to assess potential sources of heterogeneity and study stability. Finally, for rs4532 locus of $D R D 1$ gene, it was the first time that its association with the occurrence of hypertension was evaluated. To some degree, our study could provide a more precise assessment 

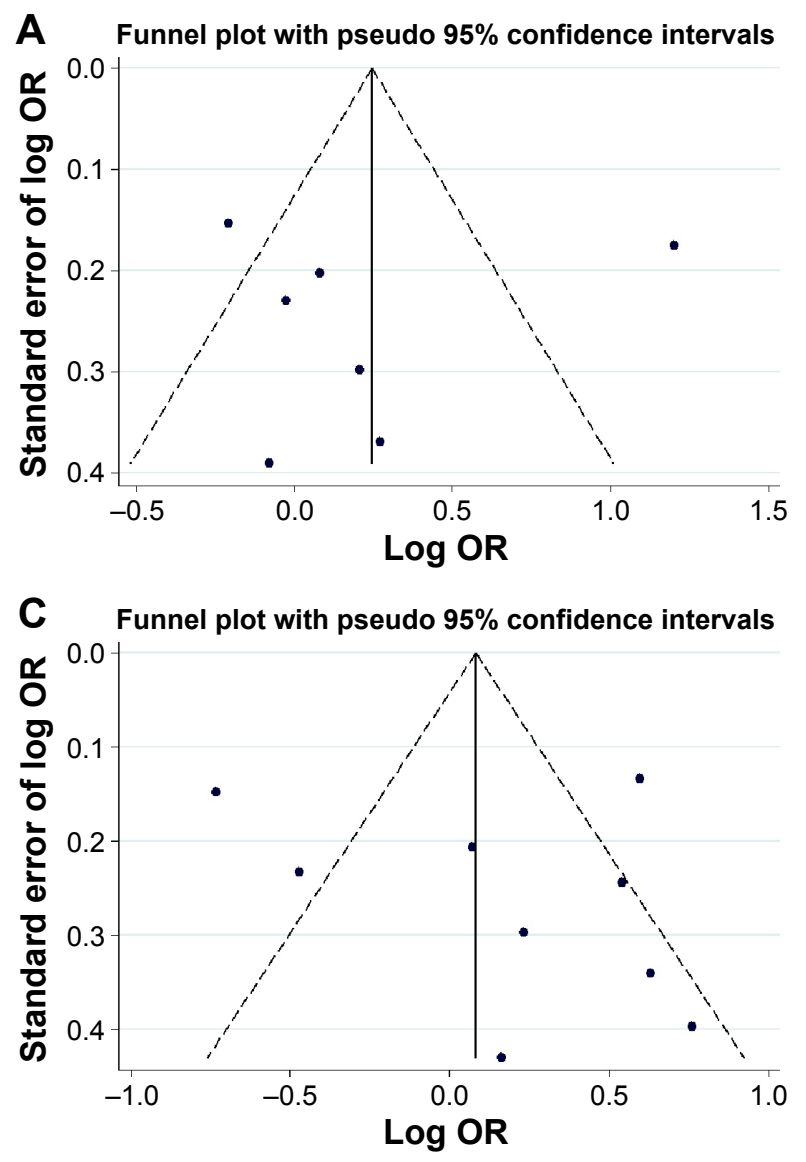
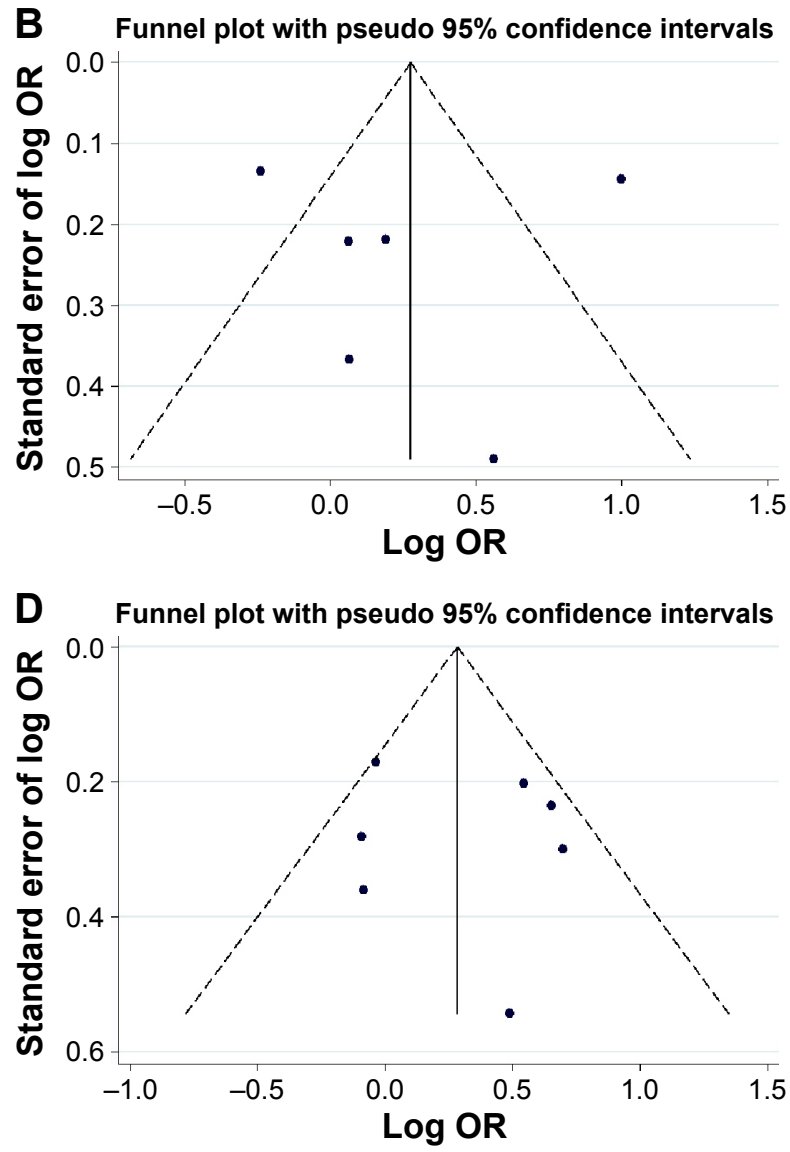

Figure 5 Funnel plot analysis on the detection of publication bias in the association between GRK4 and DRD / gene polymorphisms and hypertension. Notes: (A) For GRK4 rs I80I058 (GRK4 A486V); (B) for GRK4 rs2960306 (GRK4 R65L); (C) for GRK4 rs I 024323 (GRK4 AI42V); (D) for DRDI rs4532. Abbreviation: OR, odds ratio.

of the association between GRK4 gene and hypertension on account of the aforementioned points.

Obviously, it is reasonable that the participation degree of the hereditary factor is distinct among various ethnic populations. ${ }^{46}$ Actually, the frequency distributions of many loci are also not the same in different ethnicities. Thus, it could well be explained that rs 1024323 polymorphism was associated with hypertension in Caucasians but not in East Asians and Africans, and the association between rs4532 and hypertension was observed in East Asians but not in Caucasians. Nevertheless, the flowing and mixing of the current population will complicate the future studies and raise the rebalance of blood pressure. The variant of rs 1024323 is the allelic variant GCC to GTC, which results in the amino acid substitution of alanine to valine in residue 142. This change is related to a constitutive increase in GRK4 activity in proximal tubular cells. ${ }^{47}$ In addition, the experimental cell and animal models implicate abnormalities in dopamine receptor regulation due to receptor desensitization resulting from increased $G R K 4$ activity, then decreased $D R D$ activity and increased $A T 1$ receptor activity. ${ }^{48-50}$ For rs 4532 , located in the $5^{\prime}$ untranslated region of $D R D 1$ gene, the variant modulates the expression and stability of mRNA and influences $D R D 1$ gene expression by modifying the interaction between DNA binding domain and the regulatory elements. ${ }^{51}$

\section{Limitations}

There are several potential limitations in the present study. First, we did not assess the influence of incongruity among different definitions of hypertension patients and normotensive controls, mainly due to lacking sufficient original data. Second, rs 1024323 polymorphism was associated with hypertension in Caucasians. However, there were only two studies in a Caucasian population, so the sample size was not large enough to draw meaningful conclusions, considering that small samples with limited participants are usually accompanied by selection bias. ${ }^{52}$

\section{Conclusion}

In summary, our results suggest that rs1024323 of GRK4 and rs4532 of DRD1 loci were associated with hypertension in Caucasians and East Asians, respectively. Large 
sample epidemiological studies, especially in different ethnic populations, need to confirm the findings of our meta-analysis and investigate the latent gene-gene and/or gene-environment interactions between the susceptibility gene and hypertension.

\section{Disclosure}

The authors report no conflicts of interest in this work.

\section{References}

1. Felder RA, Jose PA. Mechanisms of disease: the role of GRK4 in the etiology of essential hypertension and salt sensitivity. Nat Clin Pract Nephrol. 2006;2(11):637-650.

2. Moore JH, Williams SM. New strategies for identifying gene-gene interactions in hypertension. Ann Med. 2002;34(2):88-95.

3. Lawes CM, Vander Hoorn S, Rodgers; International Society of H. Global burden of blood-pressure-related disease, 2001. Lancet. 2008; 371(9623):1513-1518.

4. Whelton PK, He J, Appel LJ, et al. Primary prevention of hypertension: clinical and public health advisory from The National High Blood Pressure Education Program. JAMA. 2002;288(15):1882-1888.

5. Luft FC. Twins in cardiovascular genetic research. Hypertension. 2001; 37(2 Pt 2):350-356.

6. Tanira MO, Al Balushi KA. Genetic variations related to hypertension: a review. J Hum Hypertens. 2005;19(1):7-19.

7. Nesbitt T, Coffman TM, Griffiths R, Drezner MK. Crosstransplantation of kidneys in normal and Hyp mice. Evidence that the Hyp mouse phenotype is unrelated to an intrinsic renal defect. J Clin Invest. 1992;89(5): 1453-1459.

8. Herrera M, Coffman TM. The kidney and hypertension: novel insights from transgenic models. Curr Opin Nephrol Hypertens. 2012;21(2): 171-178.

9. Belmonte SL, Blaxall BC. G protein coupled receptor kinases as therapeutic targets in cardiovascular disease. Circ Res. 2011;109(3): 309-319.

10. Yang J, Villar VA, Jones JE, Jose PA, Zeng C. G protein-coupled receptor kinase 4: role in hypertension. Hypertension. 2015;65(6): 1148-1155.

11. Zeng C, Villar VA, Eisner GM, Williams SM, Felder RA, Jose PA. $\mathrm{G}$ protein-coupled receptor kinase 4: role in blood pressure regulation. Hypertension. 2008;51(6):1449-1455.

12. Jose PA, Soares-da-Silva P, Eisner GM, Felder RA. Dopamine and $G$ protein-coupled receptor kinase 4 in the kidney: role in blood pressure regulation. Biochim Biophys Acta. 2010;1802(12):1259-1267.

13. Bhatnagar V, O'Connor DT, Brophy VH, et al. G-protein-coupled receptor kinase 4 polymorphisms and blood pressure response to metoprolol among African Americans: sex-specificity and interactions. Am J Hypertens. 2009;22(3):332-338.

14. Rayner B, Ramesar R, Steyn K, Levitt N, Lombard C, Charlton K. G-protein-coupled receptor kinase 4 polymorphisms predict blood pressure response to dietary modification in Black patients with mildto-moderate hypertension. J Hum Hypertens. 2012;26(5):334-339.

15. Vandell AG, Lobmeyer MT, Gawronski BE, et al. G protein receptor kinase 4 polymorphisms: beta-blocker pharmacogenetics and treatment-related outcomes in hypertension. Hypertension. 2012;60(4): 957-964.

16. Wagner F, Malice MP, Wiegert E, et al. A comparison of the natriuretic and kaliuretic effects of cicletanine and hydrochlorothiazide in prehypertensive and hypertensive humans. J Hypertens. 2012;30(4): 819-827.

17. Zeng C, Luo Y, Asico LD, et al. Perturbation of D1 dopamine and AT1 receptor interaction in spontaneously hypertensive rats. Hypertension. 2003;42(4):787-792.
18. Soreq H. Checks and balances on cholinergic signaling in brain and body function. Trends Neurosci. 2015;38(7):448-458.

19. Sanada H, Yoneda M, Yatabe J, et al. Common variants of the $G$ protein-coupled receptor type 4 are associated with human essential hypertension and predict the blood pressure response to angiotensin receptor blockade. Pharmacogenomics J. Epub 2015 Mar 3.

20. Xu J, Hua Q, Li DB, et al. Correlation between D1 dopamine receptor gene polymorphism and essential hypertension. Chinese Circ J. 2006;21(2):106-109.

21. Hanin G, Shenhar-Tsarfaty S, Yayon N, et al. Competing targets of microRNA-608 affect anxiety and hypertension. Hum Mol Genet. 2014; 23(17):4569-4580.

22. Bengra C, Mifflin TE, Khripin Y, et al. Genotyping of essential hypertension single-nucleotide polymorphisms by a homogeneous PCR method with universal energy transfer primers. Clin Chem. 2002;48(12): 2131-2140.

23. Williams SM, Ritchie MD, Phillips JA 3rd, et al. Multilocus analysis of hypertension: a hierarchical approach. Hum Hered. 2004;57(1): 28-38.

24. Speirs HJ, Katyk K, Kumar NN, Benjafield AV, Wang WY, Morris BJ. Association of G-protein-coupled receptor kinase 4 haplotypes, but not HSD3B1 or PTP1B polymorphisms, with essential hypertension. J Hypertens. 2004;22(5):931-936.

25. Wang Y, Li B, Zhao W, et al. Association study of G protein-coupled receptor kinase 4 gene variants with essential hypertension in northern Han Chinese. Ann Hum Genet. 2006;70(Pt 6):778-783.

26. Martinez Cantarin MP, Ertel A, Deloach S, et al. Variants in genes involved in functional pathways associated with hypertension in African Americans. Clin Transl Sci. 2010;3(6):279-286.

27. Sun CJ, Zhang WY. G protein-coupled receptor kinase 4 gene variants are not associated with preeclampsia in Northern Han Chinese. Hypertens Res. 2010;33(7):683-687.

28. Cao J, Zhu JH, Cui ZC, et al. Association study of G protein-coupled receptor kinase 4 gene polymorphism with essential hypertension. Chinese Circ J. 2007;22(3):162.

29. Kimura L, Angeli CB, Auricchio MT, et al. Multilocus family-based association analysis of seven candidate polymorphisms with essential hypertension in an african-derived semi-isolated brazilian population. Int J Hypertens. 2012;2012:859219.

30. Beige J, Bellmann A, Sharma AM, Gessner R. Ethnic origin determines the impact of genetic variants in dopamine receptor gene (DRD1) concerning essential hypertension. Am J Hypertens. 2004;17(12 Pt 1): 1184-1187.

31. Cipolletta E, Ciccarelli M, Izzo R, Finelli R, Trimarco B, Iaccarino G. A Polymorphism within the Promoter of the Dopamine Receptor D1 (DRD1-48A/G) Associates with Impaired Kidney Function in White Hypertensive Patients. Transl Med UniSa. 2012;2:10-19.

32. Yang B, Fan S, Zhi X, et al. Associations of MTHFR gene polymorphisms with hypertension and hypertension in pregnancy: a meta-analysis from 114 studies with 15,411 cases and 21,970 controls. PloS One. 2014; 9(2):e87497.

33. Liu C, Xi B. Pooled analyses of the associations of polymorphisms in the GRK4 and EMILIN1 genes with hypertension risk. Int J Med Sci. 2012;9(4):274-279.

34. Yao J, Pan YQ, Ding M, Pang H, Wang BJ. Association between DRD2 (rs1799732 and rs1801028) and ANKK1 (rs1800497) polymorphisms and schizophrenia: a meta-analysis. Am J Med Genet B Neuropsychiatr Genet. 2015;168B(1):1-13.

35. Munafo MR, Flint J. Meta-analysis of genetic association studies. Trends Genet. 2004;20(9):439-444.

36. Thakkinstian A, McElduff P, D'Este C, Duffy D, Attia J. A method for meta-analysis of molecular association studies. Stat Med. 2005; 24(9):1291-1306.

37. Higgins JP, Thompson SG, Deeks JJ, Altman DG. Measuring inconsistency in meta-analyses. BMJ. 2003;327(7414):557-560.

38. Zintzaras E, Ioannidis JP. Heterogeneity testing in meta-analysis of genome searches. Genet Epidemiol. 2005;28(2):123-137. 
39. Higgins JP, Thompson SG. Quantifying heterogeneity in a metaanalysis. Stat Med. 2002;21(11):1539-1558.

40. Egger M, Davey Smith G, Schneider M, Minder C. Bias in meta-analysis detected by a simple, graphical test. BMJ. 1997;315(7109):629-634.

41. Trikalinos TA, Salanti G, Zintzaras E, Ioannidis JP. Meta-analysis methods. Adv Genet. 2008;60:311-334.

42. Yuan GX, Ha DW. Correlation between Dopamine D1 Receptor Gene 48A/G Polymorphis mand Essential Hypertension in a Chinese Poplulation. Chinese J Hypertens. 2002;10(3):249-251.

43. Sato M, Soma M, Nakayama T, Kanmatsuse K. Dopamine D1 receptor gene polymorphism is associated with essential hypertension. Hypertension. 2000;36(2):183-186.

44. Orun O, Nacar C, Cabadak H, et al. Investigation of the association between dopamine D1 receptor gene polymorphisms and essential hypertension in a group of Turkish subjects. Clin Exp Hypertens. 2011; 33(6):418-421.

45. Arj-Ong S, Thakkinstian A, McEvoy M, Attia J. A systematic review and meta-analysis of tumor necrosis factor alpha-308 polymorphism and Kawasaki disease. Pediatr Int. 2010;52(4):527-532.
46. Pan Z, Trikalinos TA, Kavvoura FK, Lau J, Ioannidis JP. Local literature bias in genetic epidemiology: an empirical evaluation of the Chinese literature. PLoS Med. 2005;2(12):e334.

47. Jose PA, Eisner GM, Felder RA. Dopamine and the kidney: a role in hypertension? Curr Opin Nephrol Hypertens. 2003;12(2):189-194.

48. Harris RC, Zhang MZ. Dopamine, the kidney, and hypertension. Curr Hypertens Rep. 2012;14(2):138-143.

49. Zeng $C$, Wang $D$, Asico LD, et al. Aberrant $D 1$ and $D 3$ dopamine receptor transregulation in hypertension. Hypertension. 2004;43(3):654-660.

50. Sidhu A, Kumar U, Uh M, Patel S. Diminished expression of renal dopamine D1A receptors in the kidney inner medulla of the spontaneously hypertensive rat. J Hypertens. 1998;16(5):601-608.

51. Yao J, Ding M, Xing J, et al. Genetic association between the dopamine D1-receptor gene and paranoid schizophrenia in a northern Han Chinese population. Neuropsychiatr Dis Treat. 2014;10:645-652.

52. Pan YQ, Qiao L, Xue XD, Fu JH. Association between ANKK1 (rs1800497) polymorphism of DRD2 gene and attention deficit hyperactivity disorder: a meta-analysis. Neurosci Lett. 2015;590: 101-105.
Clinical Interventions in Aging

\section{Publish your work in this journal}

Clinical Interventions in Aging is an international, peer-reviewed journal focusing on evidence-based reports on the value or lack thereof of treatments intended to prevent or delay the onset of maladaptive correlates of aging in human beings. This journal is indexed on PubMed Central, MedLine,

\section{Dovepress}

CAS, Scopus and the Elsevier Bibliographic databases. The manuscript management system is completely online and includes a very quick and fair peer-review system, which is all easy to use. Visit http://www.dovepress. com/testimonials.php to read real quotes from published authors. 